\title{
Phase angle and malnutrition in Canadian community-dwelling adults
}

Peter Hannaberg MScFN RD, Justine Horne PhD (c) RD, Sylvia Rinaldi PhD (c) RD, Jason Gilliland PhD, Janet Madill PhD RD FDC

\section{Background:}

- Malnutrition remains a significant ongoing issue in Canada.

Phase angle (PhA) from bioelectric impedance analysis (BIA) may be a surrogate marker for nutritional status. ${ }^{1,2,3}$

PhA results are confounded by age, gender, and BMI. ${ }^{2}$ Thus, standardized PhA (SPhA) is a better approach for monitoring.

\section{Objectives:}

$$
\text { Phase angle }=\text { arc }- \text { tangent }\left(\frac{\text { reactance }}{\text { resistance }}\right) \times \frac{180^{\circ}}{\pi}
$$

- To determine PhA in a sample of Canadian, overweight/obese, community-dwelling adults categorized by age, gender, and BMI.

To compare PhA of our sample to a reference population matched for age, gender, and BMI.

To determine if PhA could be improved in response to a nutrition and lifestyle change intervention.

\section{Methods:}

- This study is a sub-analysis within the larger NOW Trial (NCT: 03015012).

- Overweight/obese adults attending a lifestyle counselling program in Southwestern Ontario underwent body composition analysis via BIA.

Phase angle is an output measure given by the device, and varies based on age, gender, and BMI

- Due to variability in body water, lean tissue, and adipose tissue.

- Participants' PhA values were matched for age, gender, and BMI to previously published reference values².

- Z-scores were calculated to compare our values to the reference population, then dichotomized into low or average/high SPhA groups depending on distance from the mean (i.e., $<-1 S D$, or $\geq-1 S D$, respectively).

- Statistical analysis included: means $\pm S D$, frequencies, independent and paired t-tests, and McNemar's chi-square.

\section{Results:}

Table 1. Baseline and 3 month demographics.

\begin{tabular}{|c|c|c|c|c|c|c|}
\hline & \multicolumn{3}{|c|}{ Baseline } & \multicolumn{3}{|c|}{3 Months } \\
\hline & Females $(n=63)$ & Males ( $n=9$ ) & $p$ & Females $(n=47)$ & Males $(n=8)$ & $p$ \\
\hline Age, yrs & $55.0 \pm 12.9$ & $56.7 \pm 10.5$ & 0.710 & $58.4 \pm 10.6$ & $54.0 \pm 12.3$ & 0.382 \\
\hline $\mathrm{BMI}, \mathrm{kg} / \mathrm{m}^{2}$ & $36.8 \pm 7.2$ & $35.6 \pm 6.6$ & 0.627 & $34.5 \pm 6.7$ & $33.8 \pm 7.0$ & 0.803 \\
\hline Phase angle, deg & $5.3 \pm 0.8$ & $5.6 \pm 0.6$ & 0.397 & $5.2 \pm 0.7$ & $5.7 \pm 0.9$ & 0.03 \\
\hline
\end{tabular}

Table 2. Low and average/high* phase angle matched for age, gender and BMI at baseline and 3 months.

\begin{tabular}{|c|c|c|c|c|c|c|c|}
\hline & \multicolumn{3}{|c|}{ Baseline } & \multicolumn{3}{|c|}{3 Months } & \multirow{2}{*}{$\frac{\text { McNemar } \chi^{2}}{P}$} \\
\hline & Females, $n(\%)$ & Males, $n(\%)$ & Total, n(\%) & Females, $n(\%)$ & Males, $n(\%)$ & Total, n(\%) & \\
\hline Low & $25(40)$ & $4(44)$ & $29(40)$ & $18(38)$ & $3(38)$ & $21(38)$ & 1.00 \\
\hline Average/High & $38(60)$ & $5(56)$ & $43(60)$ & $29(62)$ & $5(62)$ & $34(62)$ & 1.00 \\
\hline
\end{tabular}

*Low defined as measured phase angle $<-1$ standard deviation from the mean reference value ${ }^{2}$.

Average/High defined as measured phase angle $\geq-1$ standard deviation from the mean reference value ${ }^{2}$. Preliminary results, please do not cite.

\section{Conclusions:}

- $40 \%$ of participants are classified as malnourished at baseline and $38 \%$ at three months when using SPhA.

- Three months is likely not enough time to observe changes in PhA with this intervention.

More research is needed to determine responsiveness of this marker, and is currently ongoing.

\section{Relevance to Practice:}

- Objective markers of nutritional status important to dietitians since malnutrition comes in many forms, and influences treatment.

- SPhA provides a safe, reliable, non-invasive biomarker which may aid dietitians in screening and monitoring for malnutrition. 